\title{
LAS PLATAFORMAS TECNOLÓGICAS EN EUROPA Y ESPAÑA. UNA INICIATIVA PARA LA TRANSFERENCIA DE CONOCIMIENTO APLICADO A LA ECONOMÍA Y EL TERRITORIO.
}

\section{TECHNOLOGY PLATFORMS IN EUROPE AND SPAIN. INITIATIVE FOR APPLIED KNOWLEDGE TRANSFER TO THE ECONOMY AND THE TERRITORY}

\author{
Carlos Hugo Soria Cáceres \\ Universidad de Valladolid \\ chsoriacaceres@gmail.com
}

Recibido: febrero, 2012.

Versión final aceptada: noviembre, 2012.

PALABRAS CLAVE: innovación, plataforma, tecnología, red, territorio.

KEYWORDS: innovation, platform, technology, network, territory.

\section{RESUMEN}

La transferencia y utilización del conocimiento generado por los centros de investigación a las empresas es un elemento clave para el desarrollo económico de un país. Por ello en las últimas décadas se han puesto en marcha distintos planes de desarrollo enmarcados dentro de la llamada "Estrategia de Lisboa", plan impulsado por la Unión Europea. La estrategia pretende coordinar toda una serie de políticas estructurales para incrementar el crecimiento y basar la economía de los estados miembros en el conocimiento y la competitividad. Uno de los ejes de estos planes es la creación de las Plataformas Tecnológicas, figura bajo la que se agrupan distintos agentes vinculados a la $\mathrm{I}+\mathrm{D}+\mathrm{i}$ con intención de promocionarla y servir de punto de encuentro. Los pasos dados hasta este momento aconsejan seguir promocionando iniciativas que promocionen estas estrategias de colaboración y conocimiento a distintas escalas.

\section{ABSTRACT}

The transfer and use of knowledge generated by research centers to companies is a key element in the economic development of a country. Thus in recent decades have launched various development plans framed within the "Lisbon Strategy" plan pushed by the European Union. The strategy aims to coordinate a range of structural policies

ISSN: 0212-8594 ISSN-e: 2340-2776 № DOI: http://dx.doi.org/10.12795/rea.2011.i28.04 
Las plataformas tecnológicas en Europa y España. Una iniciativa para la transferencia de conocimiento aplicado a la economía y el territorio

to increase growth and economic base of the member states in the knowledge and competitiveness. One focus of these plans is the creation of technology platforms, figure under which different agents are grouped linked to $R$ \& $D$ with intent to promote it and serve as a meeting point. The steps taken so far are advised to continue promoting these initiatives that promote collaboration and knowledge strategies at different scales 


\section{INTRODUCCIÓN. LÍNEAS BÁSICAS DE INVESTIGACIÓN, DESARROLLO, INNOVACIÓN Y COMPETITIVIDAD.}

La conceptualización de los términos que con más frecuencia aparecen a la hora de hablar de un proceso vinculado a la $\mathrm{I}+\mathrm{D}+\mathrm{i}$ (investigación, desarrollo e innovación) suponen una primera toma de contacto fundamental a la hora de entender esta actividad, cada vez más importante en las economías modernas. El Manual de Frascati 2002 en su sexta edición, considera la investigación y el desarrollo (I+D) de manera conjunta. El resultado de ambas es un trabajo creativo destinado a incrementar el volumen de conocimientos y el uso de los mismos para derivar en nuevas aplicaciones. Ello engloba tres actividades matrices: por una parte la investigación básica, que consiste fundamentalmente en trabajos de carácter práctico o teórico iniciados con vocación de adquirir nuevos conocimientos acerca de los fundamentos de fenómenos y hechos observables, sin pensar en darles ninguna aplicación o utilización determinada; en segundo lugar la investigación aplicada, trabajos originales enfocados a la adquisición de nuevos conocimientos pero dirigidos fundamentalmente hacia un objetivo práctico específico; y por último el desarrollo experimental, trabajos basados en los conocimientos existentes, derivados de la investigación o la experiencia práctica y dirigidos a la producción de nuevos materiales, productos o dispositivos, así como a la mejora sustancial de los ya existentes.

La I+D está vinculada a un proceso de mejora y a este proceso se le ha añadido, en los últimos años, un concepto clave, la innovación, convirtiéndose así en una propuesta conjunta de Investigación, Desarrollo e innovación ( $1+D+i)$. De este modo, la innovación presupone la existencia de un elemento novedoso, que acarrea la resolución de un problema científico o tecnológico, generando la implementación de un producto o servicio significativamente mejorado. Estas actividades innovadoras pueden tener distinta naturaleza ya sean avances científicos, tecnológicos, organizacionales, financieros, comerciales etc., pero para innovar es necesario un amplio conocimiento de una necesidad, pues no todas las ideas innovadoras tienen éxito.

De este modo, la innovación, por sí sola, no garantiza necesariamente que se alcance la competitividad, ya que además de establecer metodologías y estrategias definidas para poder innovar, ésta debe acompañarse del paso previo fundamentado en la I+D. Con ello se añade un paso más, derivado, según Escorsa (1997), de un proceso en el cual a partir de una idea, invención o reconocimiento de una necesidad se desarrolla un producto, técnica o servicio útil hasta que sea comercialmente aceptado. De acuerdo con esta idea, innovar no sería más que el proceso de desarrollar algo nuevo o que no se conoce a partir del estudio metódico de una necesidad, para lograr fundamentalmente una meta económica. La motivación fundamental de la innovación es generar ideas que pueden venderse en un mercado específico, dando lugar a

ISSN: 0212-8594 ISSN-e: 2340-2776 № DOI: http://dx.doi.org/10.12795/rea.2011.i28.04

REA 28 (2011):55-69

http://www.publius.us.es/estudios_andaluces 
procesos de competitividad y apoyo desde distintas esferas administrativas y empresariales.

Por otra parte, innovación y competitividad son conceptos que suelen ir aparejados, pero no necesariamente son complementarios, de hecho, es necesario contar con todas las herramientas necesarias para que la innovación no solo tenga carácter inédito, sino que también funcione: "La innovación es el elemento clave que explica la competitividad" (Escorsa, P., 1997, 19). Se puede ser competitivo sin ser innovador (bastaría con mantener sistemas de mejora continua, de investigación y desarrollo) pero sin innovación estos procesos de I+D no llegan a ser suficientes, es decir, no son competitivos. Ello se produce cuando el mercado se encuentra saturado, cuando la demanda es alta y cuando existen necesidades que los productos o servicios existentes no logran solventar. Es ahí cuando se halla la necesidad de mejorar el producto y la cadena de producción invirtiendo en $1+D+i$, pues en este punto, la innovación se convierte en un proceso fundamental para alcanzar la competitividad, debido a que los esfuerzos por mejorar han alcanzado su límite y ya no son suficientes para seguir adelante.

\section{EL DESARROLLO DE LAS POLÍTICAS DE I+D+i.}

En el marco de la nueva economía terciarizada y de la sociedad de la información resulta fundamental ir más allá del proceso de I+D. Por esta razón, a raíz de los cambios económicos recientes las administraciones públicas, en colaboración con entidades privadas, han diseñado políticas tecnológicas destinadas a introducir nuevas actividades productivas, elevar la competitividad, la calidad y la productividad de la industria. Las distintas políticas estatales y europeas tienen como objetivo fundamental acercar este proceso de $\mathrm{I}+\mathrm{D}+\mathrm{i}$ al territorio a través de distintos programas y proyectos específicos.

En este sentido cabe destacar una idea propuesta por Méndez, R. (1997), la cual sugiere un nuevo modelo económico sobre el territorio, afectado por la creciente globalización de la economía. A partir de este proceso se generan nuevas escalas de organización industrial con efectos territoriales. Ejemplos de esta nueva economía son la deslocalización de la manufactura, la descentralización y diversificación de actividades, los nuevos factores de localización o el continuo flujo de bienes y servicios.

El resultado de todo este proceso es un espacio fragmentado en múltiples posibilidades territoriales, así como una nueva Geografía Económica con formas esencialmente urbanas surgida del agotamiento de un ciclo industrial y de la importancia que adquiere el binomio ciencia-tecnología. En este marco tiene un interés especial el territorio, sus recursos y los agentes, que con el fin de elevar el desarrollo regional están diseñando políticas industriales más o menos activas

ISSN: 0212-8594 ISSN-e: 2340-2776 № DOI: http://dx.doi.org/10.12795/rea.2011.i28.04

REA 28 (2011):55-69

http://www.publius.us.es/estudios_andaluces 
(Ontanegui, J.C., 1999). Surgen así nuevos espacios productivos ligados a I+D+i, asentados sobre parques científicos, tecnológicos o polígonos industriales verdes especialmente diseñados por y para la tecnología. A su vez, estos espacios, pretenden acercar la investigación a la producción industrial mediante la concentración de distintas empresas ligadas a este paradigma tecnológico y de innovación.

De esta manera, el binomio territorio e innovación se intenta analizar observando las conexiones entre la investigación, la actividad industrial, los agentes de innovación y las redes de transferencia tecnológicas existentes, que desde conceptos más amplios como los sistemas y redes de innovación surgen en un marco territorial en el cual múltiples actores impulsan, facilitan, restringen o incentivan el proceso. (Ontanegui, J.C., 1999). En esta red fluyen y actúan un conjunto heterogéneo de agentes con diferentes funciones (universidades, organismos públicos de investigación, agencias de desarrollo, infraestructuras de apoyo), al que se denomina Sistema CienciaTecnología-Industria configurado por los "entornos" productivo, tecnológico y científico (Nelson, E., 1993; CICYT, 1995).

Estas redes tecnológicas quedan concentradas en espacios muy específicos, que, siguiendo a autores como Cooke, P. y Morgan, K., (1994), se establecen en función de tres modelos principales. El primero denominado "dirigista" donde se concentran actividades con elevado contenido científico-tecnológico mediante la planificación de infraestructuras, modelo que ha tenido especial incidencia en países como España o Francia, donde el equipamiento tecnológico ha experimentado un gran repunte en un relativo corto espacio de tiempo. El segundo modelo se define como "espontáneo", y guarda relación con territorios donde la concentración espacial de la I+D+i está fijada por la iniciativa de actores existentes, con el apoyo entes locales o centros para la innovación.

Por último, el tercer modelo es el modelo "red", que prevé un complejo sistema de trabajo donde la presencia de un conjunto de instituciones de investigación de base y aplicada se complementa con una serie de empresas industriales que operan apoyándose en una precisa jerarquía y división de las tareas innovadoras. Este último modelo es el que siguen las políticas públicas de desarrollo de la I+D+i a nivel europeo con un apoyo de base a las Pequeñas y medianas Empresas (PYMES). En este modelo es fundamental la creación de redes de transferencia e innovación tecnológica, con el fin de introducir mecanismos de difusión que permitan un realce de la oferta y mantengan al tejido productivo en un esfuerzo de crecimiento, y de adquisición de tecnología ajustado a la demanda latente de las empresas.

ISSN: 0212-8594 ISSN-e: 2340-2776 № DOI: http://dx.doi.org/10.12795/rea.2011.i28.04

REA 28 (2011):55-69

http://www.publius.us.es/estudios_andaluces 


\section{MARCO DE ACTUACIÓN: EL ESPACIO EUROPEO DE INVESTIGACIÓN Y LOS PLANES DE INNOVACIÓN EN ESPAÑA.}

El conocimiento y la inversión en $I+D+i$ ha sido y es uno de los pilares básicos sobre el que se sustentan algunas de las principales políticas de desarrollo europeas y nacionales. La Comisión Europea (2005) considera que la inversión en conocimiento es el mejor medio de que dispone la UE para estimular el crecimiento económico y crear más empleo de mejor calidad, garantizando a un tiempo los avances sociales y la sostenibilidad medioambiental. Además de la inversión en tecnología, innovación y desarrollo, la principal función de estas políticas ha de ser la transferencia directa e indirecta de los conocimientos adquiridos, y para que esta idea se haga realidad es necesario un adecuado tejido empresarial, logístico y académico que repercuta a modo de red sobre todos y cada uno de los agentes implicados en estos procesos.

En el marco de una importancia cada vez mayor de la I+D+i, la Unión Europea y los estados miembros han puesto en marcha distintas políticas apoyadas en el denominado "triángulo del conocimiento", formado en base a los tres componentes principales que avanzan de forma consolidada: la educación, la investigación y la innovación. Fruto de estas iniciativas nació en 2005 la denominada "Estrategia de Lisboa", cuyo primer paso fue intentar convertir ese triángulo de conocimiento en un verdadero Espacio Europeo de Investigación, marco en el que además se apoyan las diferentes estrategias nacionales y locales relacionadas con esta materia.

Este espacio con vocación integradora y con vistas a estar planamente consolidado en 2014, fija la posibilidad de que los investigadores comunitarios puedan trabajar desde cualquier lugar de la UE y en el que se estreche la cooperación transfronteriza. Para este objetivo el Espacio debe crear las condiciones favorables para aumentar el impacto de las actividades de I+D en Europa y para ello la Comisión Europea, entre otras medidas, puso en marcha el VII Programa Marco de Investigación (2007-2013), enfocado hacia la consecución de los objetivos de Lisboa. Este programa, dotado con más de 50.500 millones de euros, puede considerarse la manifestación más palpable de la política europea de investigación e innovación.

En España se ha desarrollado el Plan Nacional de Investigación Científica, Desarrollo e Innovación Tecnológica (Plan Nacional de I+D+i), un instrumento de programación para la consecución de los objetivos y prioridades de la política de investigación, desarrollo e innovación tecnológica a medio plazo, según se define en la Ley de la Ciencia y en la Estrategia Nacional de Ciencia y Tecnología (ENCYT). Anualmente se elabora el Programa de Trabajo del Plan Nacional de I+D+i que, una vez aprobado, actúa como herramienta de programación a corto plazo de la política de ciencia y tecnología, como instrumento de coordinación de las actuaciones de la Administración General del Estado (AGE) y como plataforma de presentación y visualización de las

ISSN: 0212-8594 ISSN-e: 2340-2776 № DOI: http://dx.doi.org/10.12795/rea.2011.i28.04

REA 28 (2011):55-69

http://www.publius.us.es/estudios_andaluces 
actuaciones de la AGE y de las Administraciones Autonómicas en Ciencia, Tecnología e Innovación.

Este Programa de Trabajo anual incluye información sobre el calendario previsto de convocatorias públicas, con indicación de los plazos de presentación y de resolución de propuestas, la distribución económica del presupuesto anual por áreas y programas prioritarios, los órganos de gestión de cada una de las actuaciones y los tipos de beneficiarios y sectores objeto de las ayudas. Pero además de este Plan, España cuenta con una Estrategia Estatal de Innovación que prevé el fomento y la creación de estructuras que faciliten el mejor aprovechamiento del conocimiento científico y del desarrollo tecnológico. Esta estrategia se traduce en una serie de términos cuantitativos cuyo fin es duplicar la economía de la innovación en España, o lo que es lo mismo, conseguir que en el año 2015 la inversión privada anual en I+D sea 6.000 millones de euros más que en el 2009.

Además se apuesta porque en el periodo 2010-2015 se haya duplicado el número de empresas dedicadas a la I+D+i de manera que se incorporen 40.000 empresas más al tejido industrial y que éstas absorban un incremento de medio millón de empleos en este mismo período. Para lograr estos objetivos, la Estrategia Estatal de Innovación consta de cinco pilares fundamentales: la generación de un entorno proclive a la innovación, fomento de la innovación desde la demanda pública, proyección internacional, fortalecimiento de la cooperación territorial y capital humano.

\section{LAS PLATAFORMAS TECNOLÓGICAS EUROPEAS. UN COMPLETO MODELO DE TRANSFERENCIA DE CONOCIMIENTOS E INNOVACIÓN.}

En este contexto de desarrollo e innovación tanto en Europa como en España, uno de los actores que sin duda se presentan como protagonistas son las plataformas tecnológicas, que constituyen además un importante avance con el que abordar alguno de los principales retos económicos, tecnológicos o sociales a los que se enfrentan estos territorios. Las plataformas tecnológicas genéricamente consisten en "agrupaciones de entidades interesadas en un sector concreto, lideradas por la industria, con el objetivo de definir una Agenda Estratégica de Investigación (siglas en inglés: SRA) sobre temas estratégicamente importantes y con una gran relevancia social, en los cuales lograr los objetivos europeos de crecimiento, competitividad y sostenibilidad depende de los avances tecnológicos y de investigación a medio y largo plazo"(Comisión Europea, 2005b). El objetivo principal de las plataformas tecnológicas es definir las prioridades, calendarios y dotaciones económicas en un conjunto de campos de alta relevancia social así como alcanzar los objetivos de crecimiento, competitividad y desarrollo sostenible antes mencionados y establecidos por la Unión Europea tanto a medio como a largo plazo.

ISSN: 0212-8594 ISSN-e: 2340-2776 № DOI: http://dx.doi.org/10.12795/rea.2011.i28.04

REA 28 (2011):55-69

http://www.publius.us.es/estudios_andaluces 
La creación de una plataforma tecnológica supone un ejercicio de colaboración institucional, empresarial y civil. En primera instancia se hace necesario reunir todos los agentes claves del sector, principalmente industria (grande o PYME), entidades relacionadas con la transferencia de tecnología o desarrollo comercial de tecnologías y Administraciones Públicas. También son agentes importantes dentro de las Plataformas Tecnológicas los promotores y consumidores de tecnologías, Institutos de investigación y comunidad académica (especialmente para fomentar la relación industria/universidad), la comunidad financiera (bancos privados, Fondo Europeo de Inversiones, etc.) y por último la sociedad civil, incluyendo usuarios y consumidores, asegurando que las agendas de investigación incluyen a los consumidores futuros.

Las principales actividades que se desarrollan en las Plataformas tecnológicas son servicios científicos de apoyo a la investigación, desarrollo tecnológico (puesta a punto de nuevas metodologías) así como la participación en proyectos de investigación aplicada. En general están concebidas como una combinación de empresas apoyadas en centros tecnológicos y universidades, definiendo la hoja de ruta de la investigación en temáticas concretas, y se muestran como un elemento clave en la I+D+i.

La primera de estas plataformas se estableció en el sector de la aeronáutica en el año 2001, pero desde ese momento el concepto ha evolucionado mucho. Por lo general, las Plataformas gozan de un amplio grado de libertad por lo que han surgido y han evolucionado de maneras muy heterogéneas dando lugar a modelos organizativos distintos, los cuales han adquirido un papel clave para asegurar la máxima participación de la industria europea. A pesar de la heterogeneidad de participantes que conforman su estructura funcional, es necesario destacar que en la filosofía de las Plataformas Tecnológicas el liderazgo corresponde a la industria.

También es importante señalar que la Comisión Europea, si bien goza de una importancia reconocida, no organiza, controla ni dirige las Plataformas Tecnológicas, pues actúa simplemente como agente orientador, guiándolas en lo que sea necesario. La Comisión Europea no es la propietaria de las plataformas y tampoco dirige la forma en que éstas desarrollan sus actividades, sino que propicia un enfoque de colaboración liderado por la industria para definir las necesidades de investigación a medio y largo plazo. Por eso, las plataformas tecnológicas deben entenderse como un reto para la propia industria que ha de organizarse convenientemente con el objetivo de definir sus necesidades que desde las capacidades existentes o que se deban crear en materia de investigación y desarrollo tecnológico que permita alcanzar un mercado futuro altamente competitivo y globalizado.

Como toda iniciativa, las plataformas tecnológicas implican un conjunto de oportunidades que tienen que ver fundamentalmente con una mayor cooperación entre los agentes implicados en la I+D+i. Ello puede dar lugar a una mejor orientación de los esfuerzos fragmentados en este área hacia la competitividad europea con lo que

ISSN: 0212-8594 ISSN-e: 2340-2776 № DOI: http://dx.doi.org/10.12795/rea.2011.i28.04

REA 28 (2011):55-69

http://www.publius.us.es/estudios_andaluces 
se exige una mayor coordinación de las políticas y actividades europeas, nacionales y regionales de $\mathrm{I}+\mathrm{D}+\mathrm{i}$ así como una mejor implicación de los mercados europeos del capital. En la actualidad las Plataformas Tecnológicas europeas alcanzan la treintena, albergando en ellas agentes económicos europeos en diversos ámbitos, cuyo objetivo es posicionarse de forma ventajosa de cara al VII Programa Marco. Esta posición ventaja les situará frente a una gran oportunidad de cara a lograr una parte importante de los recursos financieros del mismo se destinen desde un principio a dichas iniciativas.

\section{LAS PLATAFORMAS TECNOLÓGICAS ESPAÑOLAS.}

Al abrigo de la política europea y de dos planes de desarrollo tecnológico nacionales (Plan Nacional de I+D+i 2004-2007 y el plan INGENIO 2010) se empezaron a crear las bases para poner en marcha las Plataformas Tecnológicas Españolas. Estas plataformas nacionales aparecen generalmente como nodos de las europeas, complementando a éstas, pero también es frecuente encontrar que se desenvuelven de manera independiente como iniciativa de la industria española, sin existir equivalente europeo en el ramo. De alguna manera, las Plataformas Tecnológicas Españolas suponen un interesante y exitoso instrumento de refuerzo y complemento de las europeas, a la vez que permiten encaminar esfuerzos hacia un escenario más comprometido, planificado y estructurado de la innovación en el ámbito nacional.

La filosofía de las Plataformas tecnológicas españolas se sitúa en la base de sus homólogas europeas, pero cuentan con un acicate diferenciador. En España existe un problema estructural de la I+D+i española basado en el pequeño número de grandes empresas españolas capaces de asumir un papel de liderazgo a escala europea. Además, como señala Gascón (2006) las referencias oficiales indican que España se encuentra aproximadamente en la mitad de inversión relativa en $\mathrm{I}+\mathrm{D}+\mathrm{i}$ con respecto a la media Europea, ya que apenas se acerca al 1\% del PIB. En Europa este porcentaje alcanza el $2 \%$, dato en cualquier caso aún lejos del objetivo de la Estrategia de Lisboa, fijado en el $3 \%$. Por otro lado, el perfil de la I+D+i española indica una preponderancia de la inversión pública, cuando Europa marca que la inversión del sector privado llegue a los $2 / 3$ de la inversión en $1+D+i$.

Con estos datos, lo que parece claro es que, para seguir siendo competitiva, la industria española, como la europea, necesita especializarse en áreas de alta tecnología, incrementando la inversión en investigación y mejorando la coordinación entre los agentes relevantes y elevando el contenido tecnológico de la actividad industrial. Por ello las Plataformas, manteniendo su génesis y filosofía, suponen una importante herramienta dentro del marco de actuación de las políticas emanadas por la U.E. Los mecanismos de actuación propios de las Plataformas Tecnológicas permiten la participación de industrias españolas en los grupos de alto nivel $y$, sobre todo,

ISSN: 0212-8594 ISSN-e: 2340-2776 № DOI: http://dx.doi.org/10.12795/rea.2011.i28.04

REA 28 (2011):55-69

http://www.publius.us.es/estudios_andaluces 


\section{Carlos Hugo Soria Cáceres}

Las plataformas tecnológicas en Europa y España. Una iniciativa para la transferencia de conocimiento aplicado a la economía y el territorio

fomentar la participación de la industria española en sub-áreas industriales de su interés que se definan en las Agendas Estratégicas de Investigación.

Cuadro 1. Plataformas tecnológicas europeas.

\begin{tabular}{|c|c|c|c|c|}
\hline Energía & Alta tecnología & Medio Ambiente & Producción y procesos & Transporte \\
\hline $\begin{array}{l}\text { European Biofuels } \\
\text { Technology Platform }\end{array}$ & $\begin{array}{l}\text { Embedded Computing } \\
\text { Systems - ARTEMIS }\end{array}$ & $\begin{array}{l}\text { Farm Animal Breeding } \\
\text { and Reproduction } \\
\text { Technology Platform }\end{array}$ & $\begin{array}{l}\text { European Construction } \\
\text { Technology Platform - ECTP }\end{array}$ & $\begin{array}{l}\text { Advisory Council for } \\
\text { Aeronautics Research } \\
\text { in Europe - ACARE }\end{array}$ \\
\hline \multirow[t]{2}{*}{$\begin{array}{l}\text { European Technology } \\
\text { Platform for the } \\
\text { Electricity Networks of } \\
\text { the Future - } \\
\text { SmartGrids }\end{array}$} & \begin{tabular}{|l|} 
European \\
Nanoelectronics \\
Initiative Advisory \\
Council - ENIAC
\end{tabular} & $\begin{array}{l}\text { Forest based sector } \\
\text { Technology Platform - } \\
\text { Forestry }\end{array}$ & $\begin{array}{l}\text { European Steel Technology } \\
\text { Platform - ESTEP }\end{array}$ & $\begin{array}{l}\text { European Rail Research } \\
\text { Advisory Council - } \\
\text { ERRAC }\end{array}$ \\
\hline & Robotics-EUROP & Food for Life - Food & Future Textiles and Clothing & $\begin{array}{l}\text { Waterborne ETP - } \\
\text { Waterborne }\end{array}$ \\
\hline $\begin{array}{l}\text { European Technology } \\
\text { Platform for Wind } \\
\text { Energy - TPWind }\end{array}$ & $\begin{array}{l}\text { Converged fixed and } \\
\text { Wireless } \\
\text { Communication } \\
\text { Networks - Net!Works }\end{array}$ & $\begin{array}{l}\text { Nanotechnologies for } \\
\text { Medical Applications - } \\
\text { NanoMedicine }\end{array}$ & $\begin{array}{l}\text { Future Manufacturing } \\
\text { Technologies - Manufuture }\end{array}$ & $\begin{array}{l}\text { European Road } \\
\text { Transport Research } \\
\text { Advisory Council - } \\
\text { ERTRAC }\end{array}$ \\
\hline $\begin{array}{l}\text { Zero Emission Fossil } \\
\text { Fuel Power Plants - } \\
\text { ZEP }\end{array}$ & $\begin{array}{l}\text { NEM initiative - the } \\
\text { European Thechnology } \\
\text { Platform where New } \\
\text { Media Content and } \\
\text { Networks meet }\end{array}$ & $\begin{array}{l}\text { European Technology } \\
\text { Platform for Global } \\
\text { Animal Health - ETPGAH }\end{array}$ & $\begin{array}{l}\text { European Technology Platform } \\
\text { on Sustainable Mineral } \\
\text { Resources - ETP SMR }\end{array}$ & $\begin{array}{l}\text { European Space } \\
\text { Technology Platform - } \\
\text { ESTP }\end{array}$ \\
\hline $\begin{array}{l}\text { Sustainable Nuclear } \\
\text { Technology Platform - } \\
\text { SNETP }\end{array}$ & \begin{tabular}{|l|} 
Networked European \\
Software and Services \\
Initiative - NESSI
\end{tabular} & $\begin{array}{l}\text { Plants for the Future - } \\
\text { Plants }\end{array}$ & $\begin{array}{l}\text { Water Supply and Sanitation } \\
\text { Technology Platform - WSSTP }\end{array}$ & \\
\hline $\begin{array}{l}\text { Photovoltaics - } \\
\text { Photovoltaics }\end{array}$ & \begin{tabular}{|l|} 
European Technology \\
Platform on Smart \\
Systems Integration - \\
EPoSS \\
\end{tabular} & & $\begin{array}{l}\text { Sustainable Chemistry- } \\
\text { SusChem }\end{array}$ & \\
\hline \multirow[t]{3}{*}{$\begin{array}{l}\text { Renewable Heating \& } \\
\text { Cooling - RHC }\end{array}$} & & & $\begin{array}{l}\text { Advanced Engineering } \\
\text { Materials and Technologies }\end{array}$ & \\
\hline & \begin{tabular}{|l} 
Photonics21- \\
Photonics
\end{tabular} & & $\begin{array}{l}\text { Industrial Safety ETP - } \\
\text { IndustrialSafety }\end{array}$ & \\
\hline & $\begin{array}{l}\text { Integral Satcom } \\
\text { Initiative - ISI }\end{array}$ & & & \\
\hline
\end{tabular}

Fuente: http://cordis.europa.eu

ISSN: 0212-8594 ISSN-e: 2340-2776 № DOI: http://dx.doi.org/10.12795/rea.2011.i28.04

REA 28 (2011):55-69

http://www.publius.us.es/estudios_andaluces 
A este respecto, en las Conclusiones de la Conferencia "Plataformas tecnológicas europeas: Un camino hacia el futuro de la competitividad europea", se señalaba que "la implicación española en plataformas tecnológicas conlleva una serie de esfuerzos específicos, que deben ser abordados. Estos esfuerzos pasan por la implementación plausible de los intereses industriales españoles a través de diferentes iniciativas: la participación en el diseño de las Agendas de Investigación Estratégicas Europeas, el un análisis de las mismas o bien en el Plan Nacional Español de I+D+i". Una tarea esencial de las plataformas españolas consistirá, pues, en definir su propio escenario de referencia y el correspondiente conjunto de actividades estratégicas de I+D, encajándolas, en la medida de lo posible, en el escenario europeo.

Las plataformas tecnológicas nacieron en España durante el año 2005, al amparo de las Agendas Estratégicas de Investigación, planes que definen las principales potencialidades de las industrias nacionales en sectores específicos. De este modo se puede diagnosticar dónde tiene relevancia y fuerza la industria española, además de beneficiar y asesorar la actuación de las Plataformas españolas. Las Agendas, por otra parte, también permiten una estrecha coordinación con las Plataformas Tecnológicas Europeas. Por encima de las expectativas iniciales, puede considerarse que las Plataformas Tecnológicas españolas son un elemento clave e innovador en la cooperación entre diferentes agentes, ya primera vez se puede afirmar que hay un mecanismo de cooperación efectivo y estable en el tiempo que incluye la cooperación público-privada como un eje principal.

El modelo español de plataformas tecnológicas está siendo muy valorado e imitado en varios países europeos (son los casos de Italia, Eslovenia, Polonia,...) y se puede considerar, sin miedo a equivocarse, que el mapa de plataformas es suficientemente estable y variado como para que el futuro de estos entes sea prometedor. Esta afirmación se apoya en el "Informe de Plataformas Tecnológicas 2009" que medía el impacto de las plataformas tecnológicas españolas sobre la economía en base a tres ejes: las actuaciones de la administración estatal en Plataformas Tecnológicas, la traslación de la evaluación europea al caso español y el estudio de los casos más relevantes.

En este informe se destaca el "excepcional" impacto de las Plataformas Tecnológicas Españolas desde su creación, pues en poco tiempo las plataformas han constituido redes de cooperación científica, además de posicionarse como un lugar de encuentro para todos los actores del sistema español (empresas, investigadores, centros tecnológicos y de investigación, administraciones públicas). A partir de estas iniciativas las plataformas permiten realizar recomendaciones de acciones y actuaciones para reforzar sectores estratégicos tanto en España como en Europa, no obstante es muy posible que se produzcan cambios que tienen que ver con la evolución de la propia dinámica económica e industrial de los sectores productivos. Así puede darse la

ISSN: 0212-8594 ISSN-e: 2340-2776 № DOI: http://dx.doi.org/10.12795/rea.2011.i28.04

REA 28 (2011):55-69

http://www.publius.us.es/estudios_andaluces 
creación de nuevas plataformas en área emergentes o bien la fusión de plataformas en sectores en evolución dentro de diferentes estrategias de fortalecimiento y visión ambiciosa. También, evidentemente, puede darse el caso de desaparición de otras que no han cumplido sus expectativas.

Después de más de siete años de funcionamiento de algunas plataformas tecnológicas españolas, el impacto en el sector que representan o en el área tecnológica en la que han surgido es evidente. Como principales beneficios de las actuaciones se destaca una mejor orientación de la I+D, una mayor colaboración y coordinación entre los participantes y las políticas públicas así como una mayor implicación del capital privado en la financiación de la I+D.

\section{CONCLUSIONES.}

La Estrategia de Lisboa pretendía establecer un compromiso de crecimiento a todos los niveles en Europa. Las actuaciones debían servir para alcanzar el equilibrio entre el crecimiento económico, la competitividad y el empleo, además de servir de modelo de impulso hacia sostenibilidad social y medioambiental. Pretendía pues un crecimiento conjunto y equitativo de todos los sectores económicos, siempre teniendo como prioridad aquellos relacionados con la $1+D+i$, donde prima el desarrollo de la productividad y el valor añadido de sus productos y servicios la economía basada en el conocimiento y la competitividad. De este modo, cualquier territorio que quiera poseer una economía verdaderamente competitiva y basada en el conocimiento, debe contar con apoyos públicos y privados a distintas escalas, además de invertir per se recursos económicos y materiales para generar dicho conocimiento a través de la investigación, difundirlo por medio de la formación y aplicarlo gracias a la innovación.

Recientemente se ha propuesto a los países miembros que, para 2020, inviertan un 3\% del PIB en I+D ( $1 \%$ financiación pública y $2 \%$ inversión del sector privado) y se calcula que con esta medida se pueden crear en torno a 3,7 millones de puestos de trabajo y aumentar el PIB anual en cerca de 800.000 millones de euros. Quizá sea una afirmación demasiado ambiciosa en estos tiempos de inestabilidad a todos los niveles, pero al menos la premisa se considera un primer impulso dentro de unos presupuestos donde en el ámbito europeo apenas destina un $2 \%$ de su PIB a la investigación (Eurostat) y el desarrollo (frente al 2,59 \% de los EE. UU., el 3,12 \% de Japón). La distancia que separa hoy día a los EE.UU. de la UE puede cifrarse en unos $130 \mathrm{mil}$ millones de euros al año (atribuibles en un $80 \%$ a la diferencia de gasto en investigación y desarrollo del sector privado).

ISSN: 0212-8594 ISSN-e: 2340-2776 № DOI: http://dx.doi.org/10.12795/rea.2011.i28.04

REA 28 (2011):55-69

http://www.publius.us.es/estudios_andaluces 
Carlos Hugo Soria Cáceres

Las plataformas tecnológicas en Europa y España. Una iniciativa para la transferencia de conocimiento aplicado a la economía y el territorio

Cuadro 2. Plataformas tecnológicas españolas.

\begin{tabular}{|c|c|c|c|}
\hline $\begin{array}{l}\text { ALIMENTACIÓN, } \\
\text { AGRICULTURA Y PESCA }\end{array}$ & MEDIOAMBIENTE & TRANSPORTE & NANOTECNOLOGÍA \\
\hline $\begin{array}{l}\text { Plataforma Tecnológica } \\
\text { Española Food for Life - } \\
\text { Spain }\end{array}$ & $\begin{array}{l}\text { Plataforma Tecnológica } \\
\text { Española de Química } \\
\text { Sostenible }\end{array}$ & $\begin{array}{l}\text { Plataforma Tecnológica } \\
\text { Ferroviaria Española. } \\
\text { PTFE }\end{array}$ & $\begin{array}{l}\text { Plataforma } \\
\text { Tecnológica Española } \\
\text { de Materiales } \\
\text { Avanzados y } \\
\text { nanomateriales. }\end{array}$ \\
\hline $\begin{array}{l}\text { Plataforma Tecnológica } \\
\text { Española de la Pesca y } \\
\text { Acuicultura }\end{array}$ & $\begin{array}{l}\text { Plataforma Tecnológica } \\
\text { Española del Agua y del } \\
\text { Riego }\end{array}$ & $\begin{array}{l}\text { Plataforma Tecnológica } \\
\text { Marítima. PTM }\end{array}$ & $\begin{array}{l}\text { Plataforma } \\
\text { Tecnológica Española } \\
\text { Impresión e Industrias } \\
\text { Afines. 3NEO }\end{array}$ \\
\hline $\begin{array}{l}\text { Plataforma Tecnológica } \\
\text { Española de Agricultura } \\
\text { Sostenible }\end{array}$ & $\begin{array}{l}\text { Plataforma Tecnológica } \\
\text { Española de Tecnologías } \\
\text { Ambientales. PLANETA }\end{array}$ & $\begin{array}{l}\text { Plataforma Tecnológica } \\
\text { en Logística Integral. } \\
\text { LOGISTOP }\end{array}$ & $\begin{array}{l}\text { SECTORES } \\
\text { INDUSTRIALES }\end{array}$ \\
\hline $\begin{array}{l}\text { Plataforma Tecnológica } \\
\text { del Vino }\end{array}$ & $\begin{array}{l}\text { Plataforma Tecnológica } \\
\text { Española de Protección } \\
\text { de la Costa y del Medio } \\
\text { Marino (PROTECMA) }\end{array}$ & $\begin{array}{l}\text { Plataforma Tecnológica } \\
\text { Española de la } \\
\text { Carretera }\end{array}$ & $\begin{array}{l}\text { Plataforma } \\
\text { Tecnológica del } \\
\text { Acero-PLATEA }\end{array}$ \\
\hline $\begin{array}{l}\text { BIOTECNOLOGÍA Y } \\
\text { SALUD }\end{array}$ & ENERGIA & & FOTONICA 21 \\
\hline $\begin{array}{l}\text { Plataforma Tecnológica } \\
\text { Española "Medicamentos } \\
\text { Innovadores" }\end{array}$ & $\begin{array}{l}\text { Plataforma Tecnológica } \\
\text { Española del Hidrógeno y } \\
\text { de las Pilas de } \\
\text { Combustible }\end{array}$ & $\begin{array}{l}\text { Plataforma Tecnológica } \\
\text { Española del } \mathrm{CO} 2\end{array}$ & $\begin{array}{l}\text { Plataforma } \\
\text { Tecnológica Española } \\
\text { del Sector Turístico. } \\
\text { THINKTUR }\end{array}$ \\
\hline $\begin{array}{l}\text { Plataforma Tecnológica } \\
\text { Española de Sanidad } \\
\text { Animal. Vet+i }\end{array}$ & $\begin{array}{l}\text { Plataforma Española de } \\
\text { Redes Eléctricas. } \\
\text { FUTURED }\end{array}$ & $\begin{array}{l}\text { Plataforma Tecnológica } \\
\text { Española de Eficiencia } \\
\text { Energética }\end{array}$ & $\begin{array}{l}\text { Plataforma } \\
\text { Tecnológica Española } \\
\text { de Construcción }\end{array}$ \\
\hline $\begin{array}{l}\text { Plataforma Tecnológica } \\
\text { Española de Mercados } \\
\text { Biotecnológicos }\end{array}$ & $\begin{array}{l}\text { Plataforma Tecnológica } \\
\text { del Sector Eólico - } \\
\text { REOLTEC }\end{array}$ & $\begin{array}{l}\text { Plataforma Tecnológica } \\
\text { Española de Geotermia. } \\
\text { GEOPLAT }\end{array}$ & $\begin{array}{l}\text { Plataforma } \\
\text { Tecnológica Española } \\
\text { de Seguridad } \\
\text { Industrial }\end{array}$ \\
\hline $\begin{array}{l}\text { Plataforma Tecnológica } \\
\text { Española de Tecnologías } \\
\text { Sanitarias }\end{array}$ & $\begin{array}{l}\text { Plataforma Tecnológica } \\
\text { Española de la Biomasa. } \\
\text { BIOPLAT }\end{array}$ & $\begin{array}{l}\text { Plataforma Tecnológica } \\
\text { de Energía Solar de } \\
\text { Concentración }\end{array}$ & $\begin{array}{l}\text { Plataforma } \\
\text { Tecnológica Española } \\
\text { Industria del Deporte }\end{array}$ \\
\hline $\begin{array}{l}\text { Plataforma Tecnológica } \\
\text { Española de Biotecnología } \\
\text { Vegetal }\end{array}$ & $\begin{array}{l}\text { Plataforma Tecnológica } \\
\text { Española Fotovoltaica }\end{array}$ & $\begin{array}{l}\text { Plataforma Tecnológica } \\
\text { de Energía Nuclear de } \\
\text { Fisión - CEIDEN }\end{array}$ & $\begin{array}{l}\text { Plataforma } \\
\text { Tecnológica de la } \\
\text { Industria de la ciencia } \\
\text { - INDUCIENCIA }\end{array}$ \\
\hline $\begin{array}{l}\text { Plataforma Española de } \\
\text { Nanomedicina }\end{array}$ & & & $\begin{array}{l}\text { Plataforma } \\
\text { Tecnológica Española } \\
\text { de Envase y Embalaje } \\
\text { - PACKNET }\end{array}$ \\
\hline \multicolumn{3}{|c|}{$\begin{array}{l}\text { Fuente: Ministerio de Economía y Competitividad. } \\
\text { Secretaría de Estado de Investigación, Desarrollo e } \\
\text { Innovación. }\end{array}$} & $\begin{array}{l}\text { Plataforma } \\
\text { Tecnológica de los } \\
\text { Sectores } \\
\text { Manufactureros } \\
\text { Tradicionales }\end{array}$ \\
\hline
\end{tabular}

Si en marzo de 2002 el Consejo Europeo de Barcelona estableció como objetivo el incremento del esfuerzo investigador europeo hasta situarlo en un $3 \%$ del PIB de la

ISSN: 0212-8594 ISSN-e: 2340-2776 № DOI: http://dx.doi.org/10.12795/rea.2011.i28.04

REA 28 (2011):55-69

http://www.publius.us.es/estudios_andaluces 
Unión (dos terceras partes de la inversión debería proceder del sector privado), tres años más tarde, el Consejo corroboraba ese objetivo, cuya consecución exige un esfuerzo renovado por parte de la UE y sus Estados miembros a fin de consolidar la financiación pública de la investigación, mejorar las condiciones para la inversión privada en $\mathrm{I}+\mathrm{D}+\mathrm{i}$ y el rápido aprovechamiento de sus frutos derivados.

Uno de los instrumentos que mejor puede canalizar esta inversión prevista (y deseada) en $1+D+i$ a distintas escalas son las Plataformas Tecnológicas. En la actualidad se está planificando una evaluación en profundidad de las mismas tras el Informe Ministerial de 2009, examen que debería volver a mostrar su valía y fortaleza en una época cuanto menos convulsa a la hora de hablar de inversiones en I+D+i.

Es ahora cuando estas Plataformas deben constituirse en organizaciones más estables y potentes para que ello dé lugar a la consecución de objetivos más a largo plazo. Entre estos objetivos está su internacionalización más allá del ámbito europeo y en este aspecto se puede resaltar sus avances en Iberoamérica y la cooperación con otras iniciativas europeas.

Asimismo la Administración General del Estado y las Comunidades Autónomas deben reconocerlas como interlocutor en temas que tengan que ver con la I+D. Aspectos como la regulación legal, el acceso a la sociedad civil, las políticas de innovación, la promoción de nuevos centros e instalaciones etc. son grandes desafíos que necesitan el apoyo del sector público. Por último, y con carácter práctico, es la industria la que debe recoger los beneficios generados por la Plataformas Tecnológicas. Estas actuaciones permitirán a la industria tomar la iniciativa y los resultados obtenidos dependerán, tanto de la capacidad de la industria para definir una visión y unas necesidades comunes, como de la capacidad de toda la comunidad de I+D para asumir el papel de la industria.

\section{BIBLIOGRAFÍA}

CICYT, (1996): III Plan nacional de I+D (1996-1999), Comisión Interministerial de Ciencia y Tecnología, Madrid, 352p.

CICYT (2003): Plan Nacional de I+D+i 2004-2007, Comisión Interministerial de Ciencia y Tecnología, Madrid, 118p

Comisión Europea (2005): “Construcción del Espacio Europeo de la Investigación al servicio del crecimiento", Comunicación de la Comisión, Bruselas

Comisión Europea (2005): "Plataformas tecnológicas europeas. Conocer para crecer", Oficinas de Publicaciones de la UE, Bruselas.

ISSN: 0212-8594 ISSN-e: 2340-2776 № DOI: http://dx.doi.org/10.12795/rea.2011.i28.04

REA 28 (2011):55-69

http://www.publius.us.es/estudios_andaluces 
Las plataformas tecnológicas en Europa y España. Una iniciativa para la transferencia de conocimiento aplicado a la economía y el territorio

Cooke, P.; Morgan, K (1994), "The Creative Milieu: a Regional Perspective on Innovation", in M. Dodgson, et.al.(eds), The Handbook of Industrial Innovation, pp. 5789, Edward Elgar, Cheltenham

Escorsa, P. (1997): Tecnología e innovación en la empresa. Dirección y gestión. Editorial UPC, Barcelona, 284p

FECYT (2007): Plan Nacional de Investigación Científica, Desarrollo e Innovación Tecnológica 2008-2011. Fundación Española de Ciencia y Tecnología. Madrid, 2010

FECYT (2010): Plan INGENIO, Fundación Española de Ciencia y Tecnología. En URL < www.ingenio2010.es/ >

FECYT (2002): Manual de Frascati, Fundación Española de Ciencia y Tecnología, Madrid, 282p.

Gascón, J. (2006): Un gran impulso para la 1+D+i: las plataformas tecnológicas españolas. BIT 159, pp.32-37

Méndez, R. (1997): Geografía económica. La lógica espacial del capitalismo global. Ariel, Barcelona, 400p

MICINN (2009): “Informe de Plataformas Tecnológicas 2009”, Ministerio de Ciencia e Innovación, Madrid, 84p

Nelson, E. (1993): National Innovation Systems. Oxford University Press, Oxford, 544p Ondategui, J.C (1999): "Redes de innovación y desarrollo regional en el noroeste peninsular", Revista de Estudios regionales, no 55, pp. 77-10 